\title{
Characterization of binding specificity using GST-conjugated mutant huntingtin epitopes in surface plasmon resonance (SPR)
}

\author{
Hang-Hee Cho ${ }^{1}$, Tae Hoon $\mathrm{Kim}^{2}$, Hong-Duck Kim ${ }^{3}$, Jae-Hyeon Cho ${ }^{1 *}$ \\ ${ }^{1}$ Institute of Animal Medicine, College of Veterinary Medicine, Gyeongsang National University, Jinju 52828, Korea \\ ${ }^{2}$ Department of Food Science and Biotechnology, Daegu University, Gyungsan 38453, Korea \\ ${ }^{3}$ Department of Public Health (Division of Environmental Health Science), New York Medical College, Valhalla, NY 10595-1585, USA
}

Received September 3, 2021

Revised October 29, 2021

Accepted November 24, 2021

Corresponding author:

Jae-Hyeon Cho

E-mail: jaehcho@gnu.ac.kr

https://orcid.org/0000-0003-1126-9809
Polyglutamine extension in the coding sequence of mutant huntingtin causes neuronal degeneration associated with the formation of insoluble polyglutamine aggregates in Huntington's disease (HD). Mutant huntingtin can form aggregates within the nucleus and processes of neurons possibly due to misfolding of the proteins. To better understand the mechanism by which an elongated polyglutamine causes aggregates, we have developed an in vitro binding assay system of polyglutamine tract from truncated huntingtin. We made GST-HD exon1 fusion proteins which have expanded polyglutamine epitopes (e.g., 17, 23, 32, 46, 60, 78, 81, and 94 CAG repeats). In the present emergence of new study adjusted nanotechnology on protein chip such as surface plasmon resonance strategy which used to determine the substance which protein binds in drug discovery platform is worth to understand better neurodegenerative diseases (i.e., Alzheimer disease, Parkinson disease and Huntington disease) and its pathogenesis along with development of therapeutic measures. Hence, we used strengths of surface plasmon resonance (SPR) technology which is enabled to examine binding specificity and explore targeted molecular epitope using its electron charged wave pattern in HD pathogenesis utilize conjugated mutant epitope of HD protein and its interaction whether wild type GST-HD interacts with mutant GST-HD with maximum binding affinity at $\mathrm{pH} 6.85$. We found that the maximum binding affinity of GST-HD17 with GST-HD81 was higher than the binding affinities of GST-HD17 with other mutant GST-HD constructs. Furthermore, our finding illustrated that the mutant form of GST-HD60 showed a stronger binding to GST-HD23 or GST-HD17 than GST-HD60 or GST-HD81. These results indicate that the binding affinity of mutant huntingtin does not correlate with the length of polyglutamine. It suggests that the aggregation of an expanded polyglutamine might have easily occurred in the presence of wild type form of huntingtin.

Key Words: Huntington's disease, Mutant huntingtin, Polyglutamine, Surface plasmon resonance

\section{INTRODUCTION}

Mental disorders include a group of diseases that range from depression, schizophrenia, bipolar disorder psychosis, to autism. Various social, cultural, economic, political, and environmental factors can cause mental disorders. In particular, health burden and medical cost for mental disorders including Alzheimer disease (AD), Parkinson disease (PD), and Huntington disease (HD) surged by unforeseen stress, lack of early diagnostic tools for molecular genetics, food safety, and exposure to environmental hazards like pollution (WHO, 2019).

Huntington's disease is an autosomal dominant inherited and fatal neurodegenerative disorder caused by the expansion of polyglutamine (known as polyQ) of the huntingtin protein as mutant form which resulted from CAG trinucleotide repeat at N-terminal sequence (Reddy et al, 1999; Bano et al, 2011; Dabrowska et al, 
2018). Huntington's disease is characterized by phenotypic features regarding alteration of motor neuron behavior due to dysfunction of synatptic transmission, and psychiatric aspects such as chorea and dystonia, incoordination, cognitive decline, and behavioral abnormality and a selective loss of neurons in the striatum and cortex leading to movement disorders, dementia, and eventually death due to toxic feature of CAG repeat (Vonsattel et al, 1985; Martin and Gusella, 1986; Walker, 2007; Burrus et al, 2020). The huntingtin gene has a polyglutamine stretch region translated from the polymorphic CAG repeat in its 5' coding region. The number of CAG repeats for a normal chromosome are in the range of 11 to 34 and are expanded to 40 120 in the HD chromosome (Rubinsztein et al, 1996; Koshyn and Zoghbi, 1997). In previous studies, compelling evidence demonstrated Wild-type huntingtin is involved in several essential cellular functions. It plays as an antiapoptotic protein and reduces the toxicity of mutant huntingtin in the in situ as well as in vivo (Preisinger et al, 1999; Rigamonti et al, 2000). However, the expanded polyglutamine in huntingtin is directly toxic to neuronal cells (Hackam et al, 1998; Yang et al, 2020). These polyglutamine tracts are also prone to intracellular aggregation and inclusion formation. Aggregation of mutant huntingtin has been shown to be promoted by increasing the number of polyglutamine repeats (Cooper et al, 1998; Li and XJ, 1998; Marquette et al, 2021). The mechanism causing protein aggregation is unknown, but one possibility is that the normal protein conformation is destabilized in the presence of an expanded polyglutamine tract, which leads to abnormal proteinprotein interactions and the formation of aggregates.

Currently, several studies have challenged to predict and visualize the impact of CAG molecular tract on cognitive dysfunction from the early onset symptom in mid-age patient who are suffering HD equipped with traditional imaging technique combined molecular diagnostic tool which are focusing on unfolding of connectivity clue between genetic level and phenotypic level including behavioral complications and pathogenic features and toxicities. In previous, there are many candidate proteins listed which may interact to huntingtin has been confirmed or unknown status. For example, transcriptomic factors (e.g., CA150, CBP, CtBP, HYP-A, B, C, NCOR, NF-kB, SP1, TAFII130, TBP, P53, REST-NRSE), trafficking and endocytosis molecules (e.g., HAP1, HIP1, HIP14, PACSIN1, PSD-95) signaling molecules (e.g., Calmodulin, CIP-4, FIP2, GRb2, IP31, SH3GL3, RasGAP), and metabolism-related molecules (e.g., Cystathionine $\beta$-synthase, GAPDH, HIP2). There are still unknown molecules exist due to gap of conceptual stigma or lack of detection method which region of huntingtin in HD protein are interacted specifically with those modifiers and outcome is variable as consequence of status like aggregated form and expanded pattern in the cytoplasm of neuronal cells (Li et al, 2004).

In cellular model, the study conducted by Lunkes et al examined the morphological ontology of polyglutamine aggregate and found the length of polyQ likely a fragment of a normal protein carrying 38 glutamine residues can aggregate into regular fibrils in vitro. They also demonstrated the inclusions could be degraded by ubiquitination of polyQ limited binding to the $\mathrm{N}$ terminal portion in Huntington. However, there is still an unclear relationship between epitope specificity and pathogenicity in HD (Lunkes A et al 1999).

Currently, several challenges are going on to overcome drawbacks or develop novel therapeutic alternatives which may lead to resolve issues about how to protect or counteract effectively with clearance specific binding using optimization of epitope mapping against unfolded or aggregated toxic protein in the brain or neuronal cells level. To provide some evidence-based or clues in vitro, we generated a variety of Huntington expanded CAG tracts as potential mutants and measured their binding specificity using SPR technology.

Herein, this study aims to investigate the binding affinity and its relationship between proteins containing polyglutamine traits. In this report, we have highlighted way of molecular diagnostic following development of an in vitro binding assay system that could visualize 
binding sensitivity depend on mutants' form of truncated huntingtin that consists of different lengths of polyglutamines.

\section{MATERIALS AND METHODS}

\section{Plasmid construction}

The fragments of HD gene, NcoI - ApaI fragments with CAG polyglutamine 80 repeats, were isolated from phage genomic clones derived from HD patients. To generate GST-HD exon 1 constructs, the HD exon 1 fragments were amplified by AmpliTaq Gold polymerase (Perkin Elmer, MA, USA) on a Perkin-Elmer DNA thermal cycler under following conditions: $95^{\circ} \mathrm{C}$ for 9 min, 40 cycles of $95^{\circ} \mathrm{C}(1 \mathrm{~min}), 60^{\circ} \mathrm{C}(1 \mathrm{~min})$, and $72^{\circ} \mathrm{C}(2$ min), and then $72^{\circ} \mathrm{C}$ for $10 \mathrm{~min}$ with forward (CGTGGATCCATGAAGGCCTTCGAGTCCCTC) and reverse (GATGAATTCTGGTCGGTGCAGCGGCTCCTC) primers.

The fragments were digested with BamHI and EcoRI and ligated into the same site of the expression vec-

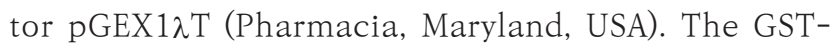
HD constructs contained 17 (GST-HD17), 23 (HD23), 32 (HD32), 46 (HD46), 60 (HD60), 78 (HD78), 81 (HD81), or 94 (HD94) CAG repeats as verified by DNA sequencing.

\section{Purification of conjugated form of GST-HD exon 1 fusion proteins}

E. coli JM109 (Toyobo, Japan) cells harboring the pGEX $1 \lambda \mathrm{T}$ expression plasmid were grown to an $\mathrm{OD}_{600}$ $\mathrm{nm}$ of 0.8 at $30^{\circ} \mathrm{C}$ in $200 \mathrm{~mL} 2 \mathrm{xYT}$ medium containing $100 \mathrm{mg} / \mathrm{mL}$ ampicillin and subsequently induced by the additional of $0.25 \mathrm{mM}$ IPTG. The cells allowed to grow for an additional $4 \mathrm{~h}$ with vigorous shaking, then collected by centrifugation (7,700 $\times \mathrm{g}$ for $10 \mathrm{~min})$, and suspended in PBS buffer ( $\mathrm{pH}$ 7.3). The suspension was sonicated with three $10-$ sec bursts. 20\% Triton X-100 was added to a final concentration of $0.1 \%$ and the resulting lysate was clarified by centrifugation $(10,000 \times \mathrm{g}$ for $30 \mathrm{~min}$ at $4^{\circ} \mathrm{C}$ ). The supernatant was incubated for
$1 \mathrm{hr}$ at room temperature with $500 \mathrm{~mL}$ of 1:1 slurry of glutathion sepharose $4 \mathrm{~B}$ beads (Sigma-Aldrich, MA, USA) that had been washed three times in $20 \mathrm{~mL}$ PBS buffer ( $\mathrm{pH}$ 7.3). The beads were poured into a small column and washed extensively PBS buffer ( $\mathrm{pH}$ 7.3). The bound fusion protein was eluted with $2 \mathrm{~mL}$ of $10 \mathrm{mM}$ reduced glutathion in $50 \mathrm{mM}$ Tris- $\mathrm{HCl}$ buffer ( $\mathrm{pH}$ 8.0). We were refined using gel filtration column more continuously to examine interaction of protein-protein. The purified fusion protein was applied to a Superdex 200 PC 3.2/30 $(2.4 \mathrm{~mL})$ column equilibrated with $10 \mathrm{mM}$ HEPES buffer ( $\mathrm{pH}$ 7.5) containing $0.15 \mathrm{M} \mathrm{NaCl}$. The chromatography column was controlled with a SMART system (Pharmacia, Maryland, USA).

\section{Immunoblot analysis}

The sample protein was denatured in a sodium dodecyl sulfate (SDS) sample buffer at $98^{\circ} \mathrm{C}$ for $5 \mathrm{~min}$, and applied to 10 20\% gradient gel (Multi Gel 10/20) purchased from Daiichi Pure Chemicals Co. (Tokyo, Japan). After electrophoresis, the proteins were transferred to polyvinylidene difluoride (PVDF) membranes (Millipore, MA, USA) and proved with anti-polyglutamine monoclonal antibody, 1C2 (Chemicon, Bedford, MA, USA). The reaction was visualized with the enhanced chemiluminescence (ECL) Western blotting system (Amersham, Arlington Heights, IL, USA). Prestained high molecular weight markers were purchased from Amersham. Protein concentrations were determined with the Coomassie Plus Protein Assay Reagent kit (Pierce Chemical Co, Dallas, Texas, USA) with bovine serum albumin as the standard protein.

\section{Surface plasmon resonance}

BIAcore 2000 system, sensor chip CM5, 10 mM HEPES buffer ( $\mathrm{pH}$ 6.85), and an amine-coupling kit containing N-hydroxysuccinimide, N-ethyl-N'-(3-diethylaminopropyl)-carbodiimide, and $1 \mathrm{M}$ ethanolaminehydrochloride, $\mathrm{pH} 8.5$, were purchased from Pharmacia 
Biosensor AB (Uppsala, Sweden). Flow cells (Fc) were prepared with the various forms: GST, GST-HD17, and GST-HD60 proteins. Immobilization of the different forms of fusion protein to the CM5 sensor chip was performed in the BIAcore system as follows. A continuous flow 10 mM HEPES buffer ( $\mathrm{pH} 7.5)$ over the sensor surface at $5 \mathrm{~mL} / \mathrm{min}$ was maintained. The carboxylated dextran matrix was activated by the injection of $35 \mathrm{~mL}$ of a solution containing 0.2 M N-ethyl-N'-(3-diethylaminopropyl)-carbodiimide and $0.05 \mathrm{M} \mathrm{N}$-hydroxysuccinimide. Next, $35 \mathrm{~mL}$ of GST, GST-HD17, and GSTHD60 (1 mM in $10 \mathrm{mM}$ sodium acetate, pH 5.0) were injected, followed by $35 \mathrm{~mL}$ of ethanolamine to block remaining $\mathrm{N}$-hydroxysuccinimide ester groups. The immobilization levels were 535.3 resonance units (RU) for GST, 1421.3 RU for GST-HD17, and 1097.2 RU for GSTHD60. To follow the interaction of the GST-HD exon 1 fusion proteins which have expanded polyglutamine tracts $(17,23,46,60$, and 81 CAG repeats) with sensor chip-coupled GST, GST-HD17, and GST-HD60, an analytical cycle consisted in the injection $15 \mathrm{~mL}$ at concentrations $10 \mathrm{mM}$ in $10 \mathrm{mM}$ HEPES buffer ( $\mathrm{pH}$ 6.85) and at a flow rate of $5 \mathrm{~mL} / \mathrm{min}$ at $25^{\circ} \mathrm{C}$. The samples were allowed to dissociate from the surface at the end of the association phase. The entire association and dissociation regions of the GST-HD exon 1 fusion protein interactions were fitted with a local fitting kinetics program supplied with the BIAcore system, from which the association rate constant, $\mathrm{ka}\left(\mathrm{in} \mathrm{M}^{-1} \mathrm{~S}^{-1}\right.$ ), the dissociation rate constant, $\mathrm{kd}$ (in $\mathrm{S}^{-1}$ ), and the equilibrium dissociation constant $\mathrm{kD}$ (in M, obtained from dividing kd by ka) were obtained. Analytical cycles were preprogrammed, and the entire analysis was completely automated.

\section{Statistical analysis}

Statistical methods Statistical analyses were performed using SPSS (SPSS, Inc., Chicago, USA). Each experiment was performed at least three times, and the results were expressed as the mean \pm standard deviation. Comparison between groups made by ANOVA variance analysis, and significant was analyzed by Ducan's multiple range tests. Differences of $P<0.05$ were considered to statistically significant.

\section{RESULTS and DISCUSSION}

First, we generated GST fusion proteins containing HD exon 1 with 17 (GST-HD17), 23 (HD23), 32 (HD32), 46 (HD46), 60 (HD60), 81 (HD81) and 94 (HD94) glutamines using recombinant molecular biotechnology to determine binding specificity among those GST conjugated fusion proteins as mutants in vitro assay. The GST-HD fusion proteins were expressed in E. coli JM109 cells and purified by affinity chromatography on glutathion sepharose 4B beads. Purified GST-HD17, GST-HD23, GST-HD32, GST-HD46, GST-HD60, GST-HD81, and GST-HD94 showed gradually increasing bands from 45 $\mathrm{kDa}$ to $65 \mathrm{kDa}$ (Fig. 1A). As we expected, mutant huntingtin proteins with the longest polyglutamine repeats (GST-HD60, GST-HD81 and GST-HD94) were aggregated, indicating that expansion of polyglutamine repeats in the huntingtin gene can induce self-aggregation in vitro. A longer exposure of the blot indicated the presence of aggregates (data not shown). These bands were also detected with the same pattern as SDS-PAGE when various protein fractions were subjected to immunoblot analysis using the anti-polyglutamine monoclonal antibody, 1C2, which specifically recognizes long polyglutamine stretches (Fig. 1B). The pattern of migration of the bands was similar in anti-GST and 1C2 antibody (Fig. 1C). All recombinant proteins migrated at a size corresponding to that predicted from their amino acid sequence. Moreover, we also detected GST-HD protein by enzyme-linked immunosorbent assay (ELISA) using the anti-polyglutamine monoclonal antibody, 1C2. These results were nearly identical to those obtained using immunoblot analysis (data not shown).

Next, we employed surface plasmon resonance, which is a powerful tool for real time measurement of direct protein-protein interactions, to investigate the interaction between the recombinant expanded GST-HD pro- 
A

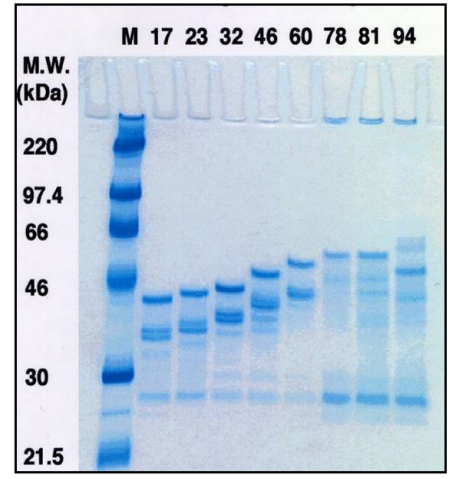

$\mathrm{B}$

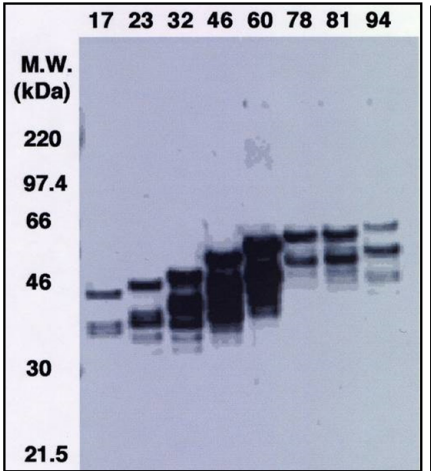

$\mathrm{C}$

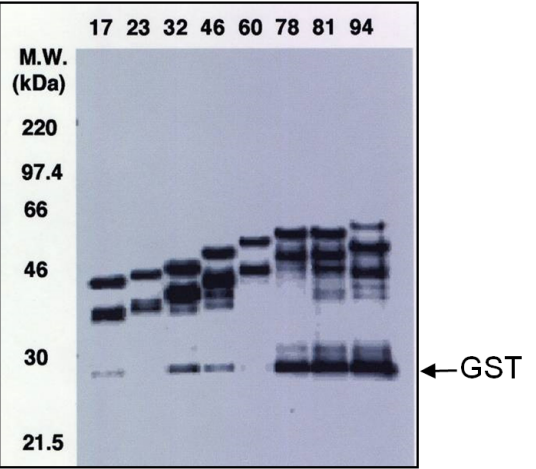

Fig. 1. SDS-PAGE and western blot analysis of purified GST and GST-HD fusion proteins. Characterization of mutant GST-HD fusion proteins and profile of expression on SDS-PAGE in Western Blot analysis. (A) The same amount of aliquots of eluates from the glutathione sepharose 4B beads column were applied to 10 20\% gradient gel SDS-PAGE and analyzed by staining with Coomassie blue R250. Lane M, size markers, Lanes 1 8 contain GST-HD17, -HD23, -HD32, -HD46, -HD60, -HD78, -HD81, and -HD94, respectively. (B) Western blot analysis with 1C2 antibody. Equimolar amounts of HD exon 1 fusion proteins were loaded and probed with anti-polyglutamine monoclonal antibody, 1C2. The numbers at the top of gel represent CAG repeats. (C) Western blots using Anti-GST monoclonal antibody. Proteins were transferred to Polyvinylidene Difluoride (PVDF) membranes and probed with anti-GST antibody.

tein and antibody. GST-HD binding experiments were performed on BIAcore X apparatus as described earlier (Sharp et al, 1995). Upon the addition of a molecule in solution to a surface bound ligand, the signal measured, which is proportional to the mass detected, provides a sensorgram, which allows to monitor the interaction.

Purified GST-HD exon 1 fusion protein in $10 \mathrm{mM}$ HEPES buffer was used to determine the affinity of the GST-HD constructs. The $\mathrm{pH}$ of the buffer was varied to screen for the best conditions of this interaction. The affinity was higher at $\mathrm{pH}$ 6.85. The aggregates were dramatically increased at lower pH 6.80 (data not shown). Therefore, all following experiments were performed in $10 \mathrm{mM}$ HEPES buffer at $\mathrm{pH}$ 6.85. In the present study, the results showed an overlay plot of net interaction curves obtained with anti-polyglutamine monoclonal antibody, 1C2, immobilized GST-HD17 and HD60 (Fig. 2). The binding affinity showed a dose-dependent increase in the concentrations of $1 \mathrm{C} 2$, which were $0.2,0.4$, $0.6,0.8$, and $1.0 \mu \mathrm{M}$, respectively.

Previous studies demonstrated that wild-type huntingtin is sequestered into aggregates by mutant huntingtin, and mutant huntingtin interferes with wild-type normal function (Busch et al, 2004). We investigated in more detail the interaction of recombinant huntingtin fragment with expanded or normal ployglutamine tracts within exon 1 of huntingtin protein. To determine the binding characteristics of normal and mutant huntingtin, the binding affinity was evaluated by calculating the interaction of GST-HD exon 1 fusion proteins (17, 23, 46, 60, and 81 glutamine repeats) with an immobilized GST-HD17 and HD60 using SPR (Fig. 3). The CAG repeat length in the normal population ranges from 10 to 35 , whereas it ranges from 36 to 121 in patients with $\mathrm{HD}$, and patients usually have an expansion of more than 60 CAG repeats. To eliminate self-aggregations before initiating the binding reaction, we investigated conditions in which expanded HD exon 1 was unable to self aggregates. Interaction of GST-HD fusion proteins $(10 \mu \mathrm{M})$ with an immobilized GST-HD17 and HD60 was studied. GST did not interact with wild type or mutant hungtingtin, indicating that GST itself cannot binds to polyglutamine tracts within exon 1 of huntingtin gene. The binding affinity of HD17 with mutant huntingtin increased with increasing polyglutamine repeats. HD17 had a higher binding affinity with HD81 than with HD46. These results indicate that the binding affinity of wild-type huntingtin with mutant huntingtin is higher 
A

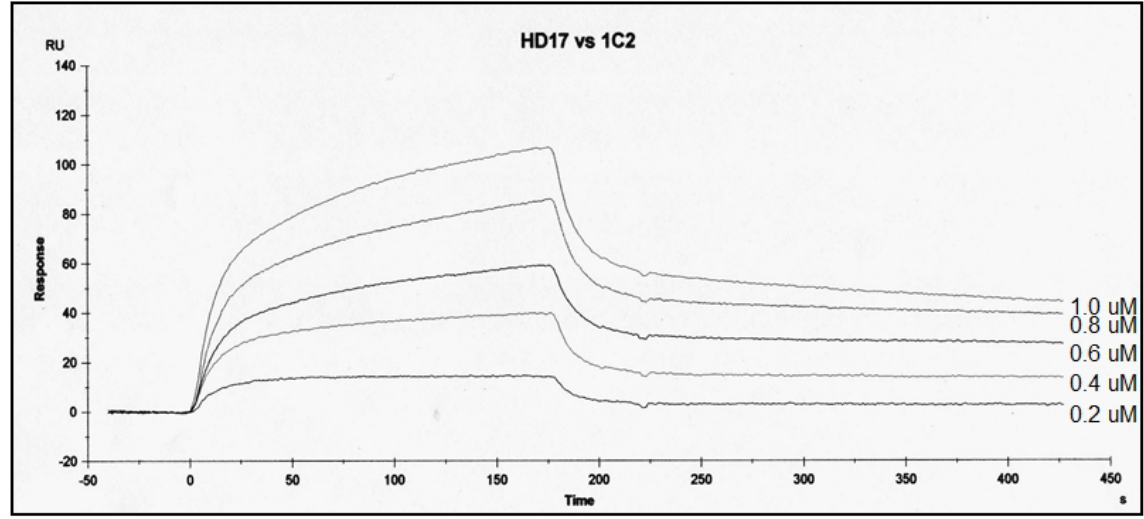

B

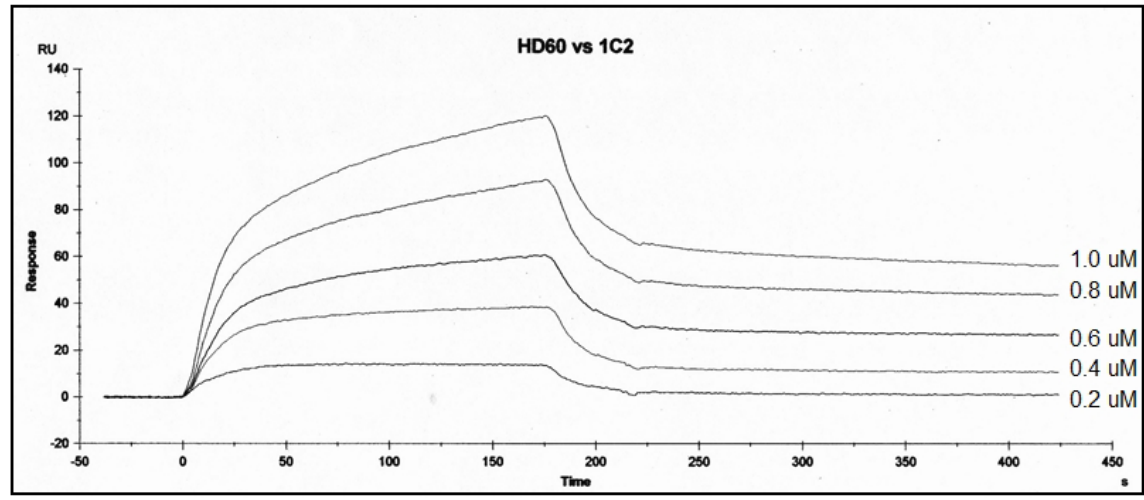

Fig. 2. Visualiztion pattern of antipolyglutamine monoclonal antibody, $1 \mathrm{C} 2$, interaction with GST-HD17 and -HD60 in surface plasmon resonance (SPR). An overlay plot of net interaction curves was obtained with anti-polyglutamine monoclonal antibody, 1C2, immobilized GSTHD17 (A) and -HD60 (B). Running buffer was $10 \mathrm{mM}$ HEPES buffer, pH7.5. Flow rate was established at $5 \mu \mathrm{L} / \mathrm{min}$. The $1 \mathrm{C} 2$ was injected at $0 \mathrm{sec}$, and the injection was terminated at $180 \mathrm{sec}$ when the buffer was allowed to flow over the sensor surface.
A

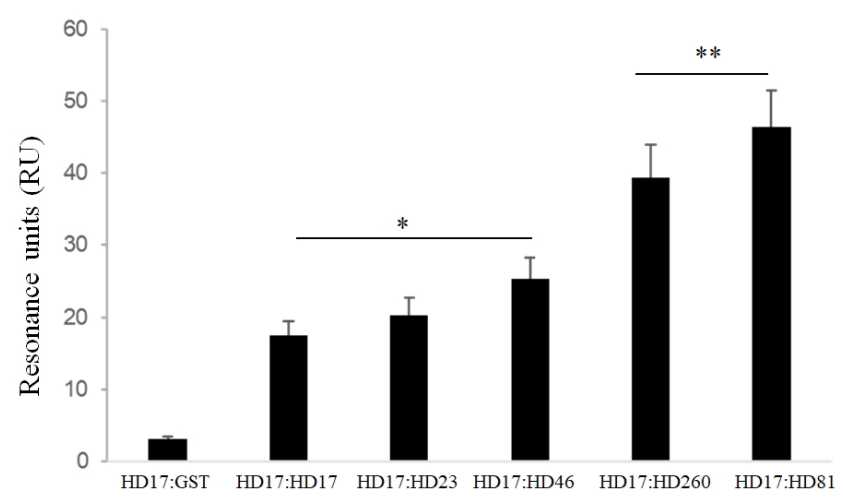

B

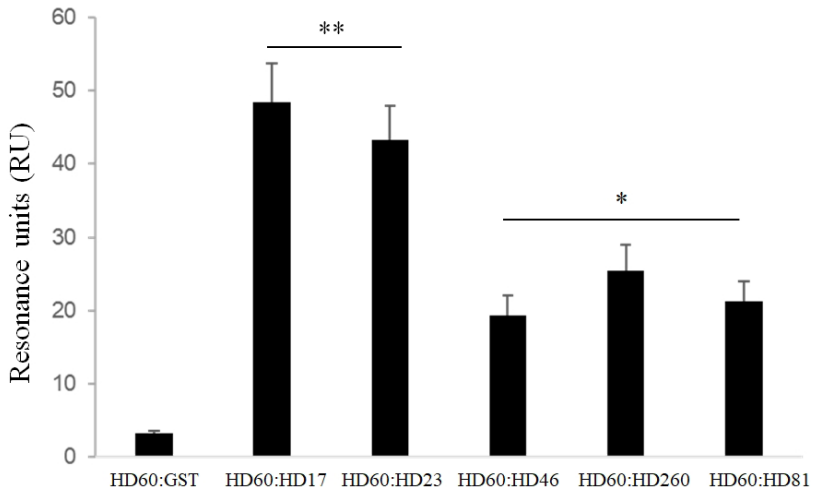

Fig. 3. Binding specificity with length of Polyglutamine utilize various GST-HD to HD17 and HD60. Plasmon resonance kinetic analysis was used to determine the binding affinity of GST-HD17 (A) or GST-HD60 (B) with $10 \mu \mathrm{M}$ GST-HD exon 1 fusion proteins that contained glutamine lengths of 17, 23, 46, 60, or 81 residues. Equimolar of GST-HD17 or GST-HD60 (1 mM in $10 \mathrm{mM}$ sodium acetate, $\mathrm{pH} 5.0)$ were applied to the sensor chip. Data are expressed as mean \pm SD for three independent assays. $* P<0.05$ compared with HD17 versus GST (control); **P<0.01 compared with HD17 versus GST.

than that of wild-type huntingtin with other normallength forms. These data are consistent with the finding that wild type huntingtin fragments with normal poly- glutamine domains do not aggregate (Busch et al, 2004). However, HD60 had a high binding affinity with HD17, but the binding affinity of HD60 with itself or other 
mutant huntingtin proteins decreased significantly. Our results suggest that the binding affinity of expanded polyglutamine huntingtin preferentially binds HD23 or HD17 over HD81. These results support the previous findings that expanded mutant polyglutamine has the potential to interact with any normal polyglutaminecontaining protein in the cells (Narain et al, 1999; Reddy et al, 1999). Moreover, wild-type huntingtin (25 length of CAGs epitope repeat) did not aggregate when transfected alone, whereas normal-length polyglutamine containing peptides were trapped in insoluble detergent-resistant aggregates when co-transfected with expanded polyglutamine tracts (Preisinger et al, 1999). The neuronal aggregates that are observed in HD are not only composed of mutant huntingtin but they are also composed of normal huntingtin, including the other proteins (Jana et al, 2000; Kim et al, 2002; Li et al, 2002; Zuccato et al, 2003). It still debatable issue remain and there is also existed an indistinguishable clinical presentation remarks in homozygote and heterozygote HD patients.

To date, there are considerable evidence rooted from tissue immunohistochemical and biochemical analyses have demonstrated that the location of wild-type huntingtin in neurons is mainly cytoplasmic (Sharp et al, 1995; Bhide et al, 1996). However, research outcome is quite variable to unveil role of the $\mathrm{N}$-terminal fragment of huntingtin that contains polyglutamine repeats forms aggregates in the nucleus and cytoplasm of affected neurons (DiFiglia et al, 1997; Martindale et al, 1998; Hackam et al, 1999; Moulder et al, 1999). Interestingly, huntingtin aggregates or protein trapped into aggregates have a different pattern in cytosol and in nucleus. Particularly, huntingtin interacts with genespecific activator protein Sp1 and selective components of the core transcription apparatus, including TFIID and TFIIF130, which induce transcriptional dysregulation implicated in HD pathogenesis (Dunah et al, 2002). For example, although soluble form of mutant huntingtin binds more tightly to Sp1 than that of aggregated huntingtin, $\mathrm{N}$-terminal mutant huntingtin in the nucleus interacts with the transcriptional factor, Sp1 or another coactivator, TAFII130 (Dunah et al, 2002; Li et al, 2002) which may disrupts the normal transcriptional machinery in regulated gene in the neurons. In contrary, several study demonstrated heterogeneity regarding huntingtin aggregates in the cytosol which are associated with a variety of cellular proteins, including ubiquitin, components of the proteasome, molecular chaperones as HSP 40 and Hsp70 and cytoskeleton proteins (Jana et al, 2000; Kim et al, 2002; Zuccato et al, 2003). Taken together, it is possible that in heterozygous HD patients wild-type huntingtin in the cytosol is aggregated with mutant huntingtin into the complexes. Conversely, homozygous patients have more nuclear aggregation of mutant huntingtin. Therefore, we suggest that wild-type huntingtin plays an important role in determining its binding affinity with mutant huntingtin and leading to aggregate formation in cytoplasmic aggregations.

In the future study, it is worth exploring the value of targeting epitope in diagnostic and therapeutic challenge following high throughput antibody production to prevent and predict neurodegenerative disease like Huntington's disease (HD). With regard to nanotechnology-driven protein binding analysis including SPR have equipped with protein on chip as well as protein array such as comparative genomic hybridization and chromatin immunoprecipitation oriented sequencing technique, it gives more comprehensive understanding of the unfolded protein as cue of pathogenic trigger in the brain health and socioeconomic impact and future needs of underserved and unmet solution demanded to community and global concern cope with potential risk surrounding environmental stressor in mental health.

Limitation of this binding specificity assay using SPR technology has shown molecular interaction in vitro level. Future study needs to develop an advanced platform to determine the specificity of HD epitope and selectivity of each epitope in cellular or in vivo model after being adopted by a functional multi-omics approach such as multi-epitope-ligand cartography in toponomics, interactomes, and fluxomics. 


\section{ACKNOWLEDGEMENTS}

This research was supported in part by Basic Science Research Program through the National Research Foundation of Korea (NRF) funded by the Ministry of Education (No. 2020R1F1A104896911).

\section{CONFLICT OF INTEREST}

No potential conflict of interest relevant to this article was reported.

\section{ORCID}

Hang-Hee Cho, https://orcid.org/0000-0003-3872-4437

Tae Hoon Kim, https://orcid.org/0000-0003-0428-2829

Hong-Duck Kim, https://orcid.org/0000-0002-6980-8687

Jae-Hyeon Cho, https://orcid.org/0000-0003-1126-9809

\section{REFERENCES}

Bano D, Zanetti F, Mende Y, Nicotera P. 2011. Neurodegenerative processes in Huntington's disease. Cell Death Dis. 10;2(11): e228.

Bhide PG, Day M, Sapp E, Schwarz C, Sheth A, Kim J, Young AB, Penney J, Golden J, Aronin N, M. DiFiglia M. 1996. Expression of normal and mutant huntingtin in the developing brain. J Neurosci. 16: 5523-35.

Burrus CJ, McKinstry SU, Kim N, Ilcim Ozlu M, Santoki AV, Fang FY, Ma A, Karadeniz YB, Worthington AK, Dragatsis I, Zeitlin S, Yin HH, Eroglu C. 2020. Striatal Projection Neurons Require Huntingtin for Synaptic Connectivity and Survival. Cell Rep. 30(3): 642-657.

Busch A, Engemann S, Lurz R, Okazawa H, Lehrach H, Wanker EE. 2003. Mutant huntingtin promotes the fibrillogenesis of wild-type huntingtin: a potential mechanism for loss of huntingtin function in Huntington's disease. J Biol Chem. 278: 41452-61.

Cooper JK, Schilling G, Peters MF, Herring WJ, Sharp
AH, Kaminsky Z, Masone J, Khan FA, Delanoy M, Borchelt DR, Dawson VL, Dawson TM, Ross CA. 1998. Truncated N-terminal fragments of huntingtin with expanded glutamine repeats form nuclear and cytoplasmic aggregates in cell culture. Hum Mol Genet. 7: 783-90.

Dabrowska M, Juzwa W, Krzyzosiak WJ, Olejniczak M. 2018. Precise Excision of the CAG Tract from the Huntingtin Gene by Cas9 Nickases. Front Neurosci. 12: 75 .

DiFiglia M, Sapp E, Chase KO, Davies SW, Bates GP, Vonsattel JP, Aronin N. 1997. Aggregation of huntingtin in neuronal intranuclear inclusions and dystrophic neurites in brain. Science. 277: 1990-3.

Dunah AW, Jeong H, Griffin A, Kim YM, Standaert DG, Hersch SM, Mouradian MM, Young AB, Tanese N, Krainc D. 2002. Sp1 and TAFII130 transcriptional activity disrupted in early Huntington's disease. Science. 296: 2238-43.

Hackam AS, Singaraja R, Wellington CL, Metzler M, McCutcheon K, Zhang T, Kalchman M, Hayden MR. 1998. The influence of huntingtin protein size on nuclear localization and cellular toxicity. J Cell Biol. 141: 1097-105.

Hackam AS, Singaraja R, Zhang T, Gan, L. Hayden MR. 1999. In vitro evidence for both the nucleus and cytoplasm as subcellular sites of pathogenesis in Huntington's disease. Hum Mol Genet. 8: 25-33.

Jana NR, Tanaka M, Wang G, Nukina N. 2000. Polyglutamine length-dependent interaction of Hsp 40 and Hsp70 family chaperones with truncated Nterminal huntingtin: their role in suppression of aggregation and cellular toxicity, Hum Mol Genet. 9: 2009-18.

Kim S, Nollen EA, Kitagawa K, Bindokas VP, Morimoto RI. 2002. Polyglutamine protein aggregates are dynamic. Nat Cell Biol. 4: 826-31.

Koshy BT, Zoghbi HY. 1997. The CAG/polyglutamine tract diseases: gene products and molecular pathogenesis. Brain Pathol. 7: 927-42.

Li SH, Cheng AL, Zhou H, Lam S, Rao M, Li H, Li XJ. 
2002. Interaction of Huntington disease protein with transcriptional activator Sp1. Mol Cell Biol. 22: $1277-87$.

Li SH, Li XJ. 1998. Aggregation of N-terminal huntingtin is dependent on the length of its glutamine repeats. Hum Mol Genet. 7: 777-82.

Li SH, Li XJ. 2004. Huntingtin-protein interactions and the pathogenesis of Huntington's disease. Trends in Genetics. 20: 146-154.

Lunkes A, Trottier Y, Fagart J, Schultz P, Zeder-Lutz G, Moras D, Mandel JL. 1999. Properties of polyglutamine expansion in vitro and in a cellular model for Huntington's disease. Philos Trans R Soc Lond B Biol Sci. 354: 1013-1019.

Marquette A, Aisenbrey C, Bechinger B. 2021. Membrane Interactions Accelerate the Self-Aggregation of Huntingtin Exon 1 Fragments in a Polyglutamine Length-Dependent Manner. Int J Mol Sci. 22(13): 6725.

Martin JB, Gusella J. 1986. Huntington's disease. Pathogenesis and management. N Engl J Med. 315; 126776.

Martindale D, Hackam A, Wieczorek A, Ellerby L, Wellington C, McCutcheon K, Singaraja RKazemiEsfarjani P, Devon R, Kim SU, Bredesen DE, Tufaro F, Hayden MR. 1998. Length of huntingtin and its polyglutamine tract influences localization and frequency of intracellular aggregates. Nat Genet. 18: 150-4.

Moulder KL, Onodera O, Burke JR, Strittmatter WJ, Jr. Johnson EM. 1999. Generation of neuronal intranuclear inclusions by polyglutamine-GFP: analysis of inclusion clearance and toxicity as a function of polyglutamine length. J Neurosci. 19: 705-15.

Narain Y, Wyttenbach A, Rankin J, Furlong RA, Rubinsztein DC. 1999. A molecular investigation of true dominance in Huntington's disease. J Med Genet. 36: $739-46$.

Preisinger E, Jordan BM, Kazantsev A, Housman D. 1999. Evidence for a recruitment and sequestration mechanism in Huntington's disease. Philos Trans R
Soc Lond B Biol Sci. 354: 1029-34.

Reddy PH, Williams M, Tagle DA. 1999. Recent advances in understanding the pathogenesis of Huntington's disease. Trends Neurosci. 22: 248-55.

Rigamonti D, Bauer JH, De-Fraja C, Conti L, Sipione S, Sciorati C, Clementi E, Hackam A, Hayden MR, Li Y, Cooper JK, Ross CA, Govoni S, Vincenz C, Cattaneo E. 2000. Wild-type huntingtin protects from apoptosis upstream of caspase-3. J Neurosci. 20: 370513.

Rubinsztein DC, Leggo J, Coles R, Almqvist E, Biancalana V, Cassiman JJ, Chotai K, Connarty M, Crauford D, Curtis A, Curtis D, Davidson MJ, Differ AM, Dode C, Dodge A, Frontali M, Ranen NG, Stine OC, Sherr M, Abbott MH, Franz ML, Graham CA, Harper PS, Hedreen JC, Jackson A, Kaplan JC, Losekoot M, MacMillan JC, Morrison P, Trottier Y, Novelletto A, Simpson SA, Theilmann J, Whittaker JL, Folstein SE, Ross CA, Hayden MR. 1996. Phenotypic characterization of individuals with 30-40 CAG repeats in the Huntington disease (HD) gene reveals HD cases with 36 repeats and apparently normal elderly individuals with 36-39 repeats. Am J Hum Genet. 59: 16-22.

Sharp AH, Love SJ, Schilling G, Li SH, Li XJ, Bao J, Wagster MV, Kotzuk JA. Steiner JP, Lo A, Hedreen J, Sisodia S, Snyder SH, Dawson TM, Ryugo DK, Ross CA. 1995. Widespread expression of Huntington' s disease gene (IT15) protein product. Neuron. 14: 1065-74.

Vonsattel JP, Myers RH, Stevens TJ, Ferrante RJ, Bird ED, Richardson Jr EP. 1985. Neuropathological classification of Huntington's disease. J Neuropathol Exp Neurol. 44; 559-77.

Walker FO, 2007. Huntington's disease. Lancet. 369 (9557): 218-28.

World Health Organization. 2019.disorders. https:// www.who.int/news-room/fact-sheets/detail/mental-disorders.

Yang H, Yang S, Jing L, Huang L, Chen L, Zhao X, Yang W, Pan Y, Yin P, Qin ZS, Tang B, Li S, Li XJ. 2020. 
Truncation of mutant huntingtin in knock-in mice demonstrates exon1 huntingtin is akey pathogenic form. Nat Commun. 11(1): 2582.

Zuccato C, Tartari M, Crotti A, Goffredo D, Valenza M, Conti L, Cataudella T, Leavitt BR, Hayden MR, Tim- musk T, Rigamonti D, Cattane E. 2003. Huntingtin interacts with REST/NRSF to modulate the transcription of NRSE-controlled neuronal genes. Nat Genet. 35: 76-83. 